\title{
Streptococcus pluranimalium
}

National Cancer Institute

\section{Source}

National Cancer Institute. Streptococcus pluranimalium. NCI Thesaurus. Code C124412.

A species of Gram-positive coccus-shaped, non-motile bacteria in the order

Lactobacillales. S. pluranimalium is positive for acid production from glucose, fructose,

and trehalose. This species is primarily responsible for infection in bovine and avian

species with only limited identification of infection in human clinical isolates. 